\title{
Suppression of feeding by intrahypothalamic implants of estradiol in male and female rats*
}

\author{
WILLIAM W. BEATTY, DENNIS A. O'BRIANT, and THOMAS R. VILBERG \\ North Dakota State University, Fargo, North Dakota 58102
}

\begin{abstract}
The effect of intrahypothalamic implants of crystalline estradiol benzoate (EB) on feeding was examined in gonadectomized male and female rats. With the exception of one animal, whose cannula terminated in the third ventricle, all sites at which EB produced substantial suppression of feeding were located in the ventromedial hypothalamic nuclei (VMH). There was no sex difference in the proportion of sites within the VMH at which EB implants suppressed feeding.
\end{abstract}

Wade and Zucker (1970) reported that implants of estradiol benzoate (EB) into the ventromedial hypothalamus (VMH) of ovariectomized female rats produced substantial suppression of feeding in most animals. At these same sites, testosterone, progesterone, or cholesterol were generally ineffective and EB implants into the lateral hypothalamus or the anterior hypothalamic preoptic region also failed to reliably suppress feeding.

Although peripheral EB treatment depresses food intake and body weight in adult rats of both sexes, females are substantially more responsive than males (Zucker, 1969). This difference is in part the result of an organizational influence of androgens during the early neonatal period, since a single injection of testosterone at this time reduces the weight-inhibiting action of adult EB treatment in females (Beatty, Powley, \& Keesey, 1970; Bell \& Zucker, 1971). Neonatal testosterone treatment also reduces the uptake of radioactive estrogen in the anterior and middle parts of the hypothalamus (Flerko, Mess, \& Illei-Donhoffer, 1969). Moreover, bilateral implants of testosterone propionate (TP) pellets into the VMH-arcuate region of neonatal female rats reproduce the anovulatory persistent estrus syndrome that results from peripheral TP treatment at the same age. Similar implants at other hypothalamic sites were generally ineffective (Nadler, 1971). Thus, one might anticipate a sex difference in the effectiveness of central EB treatment on feeding. To test this possibility, we examined the effects on feeding of EB implants into the VMH in both male and female rats.

\section{METHOD}

A complete description of the surgical procedures and construction of the cannula-electrode array is available elsewhere (O'Briant, 1974), so only a brief description will be given. Thirty-five male and 44 female Holtzman rats, 4-7 months of age, that had been gonadectomized 2-3 months earlier, were implanted with an array consisting of a single outer cannula

*This experiment is based on a portion of a thesis submitted by Dennis O'Briant in partial fulfiliment of the requirements for a MS degree in psychology at North Dakota State University. The work was supported by a NIMH Grant (MH 23172-01) to William W. Beatty. fashioned from 21-ga stainless steel tubing and two stainless steel 0 -ga insect pins insulated, except at the tip, with Expoxylite. The outer cannula was implanted so that it terminated $1.0 \mathrm{~mm}$ above the center of the right $\mathrm{VMH}$, while the lesioning electrodes were aimed bilaterally at the lateral hypothalamic (LH) feeding area. We hoped to examine the effects of unilateral LH lesions on suppression of feeding by EB implants in $\mathrm{VMH}$, but the data are too incomplete to report.

At the time of surgery, each animal was fitted with an empty 27-ga inner cannula cut to protrude $1.0 \mathrm{~mm}$ below the base of the outer cannula. This inner cannula remained in place at all times except when it was replaced with a test cannula of identical construction that was packed with crystalline EB or cholesterol (Chol) as described by Wade and Zucker (1970).

The animals were caged singly in an air-conditioned animal room maintained on a 12-h light-dark cycle. Food (powdered Purina Lab Chow) and water were freely available in each rat's home cage throughout the experiment. Following a 1-week postoperative recovery period, the stability of each rat's food intake was assessed. When each animal exhibited a variation of less than $10 \%$ in the means of two consecutive 3-day blocks, then the average daily intake for the next 5 days was used as a baseline for the suppression test. After obtaining a baseline, the effects of EB or Chol implants were assessed by replacing the empty inner cannula with another packed with the test compound for a 3-day period. Sites were rated positive when both of the following conditions were met: (1) in a minimum of two of three trials, EB implants caused at least a $15 \%$ reduction in food intake from baseline, and (2) on one trial, Chol caused less than an $8 \%$ reduction in food intake. At least 1 week for recovery intervened between testing. At the conclusion of testing, placements were verified histologically.

\section{RESULTS AND DISCUSSION}

Figure 1 summarizes the location of positive and negative sites for all animals tested. Nine of 18 males $(50 \%)$ and 10 of 24 females $(42 \%)$ with cannulae that terminated within the VMH nuclei met the criteria described above and were considered to have positive sites. An additional 17 males and 19 females had cannulae located within the hypothalamus, but outside the VMH nuclei; all of these sites were negative. One female rat with a cannula that terminated in the third ventricle posterior to the VMH also met the suppression criteria.

The present results confirm earlier findings (Wade \& Zucker, 1970) demonstrating that implants of EB into 
MALE

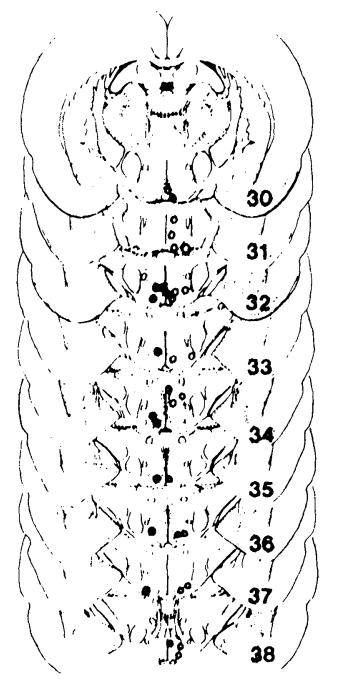

FEMALE

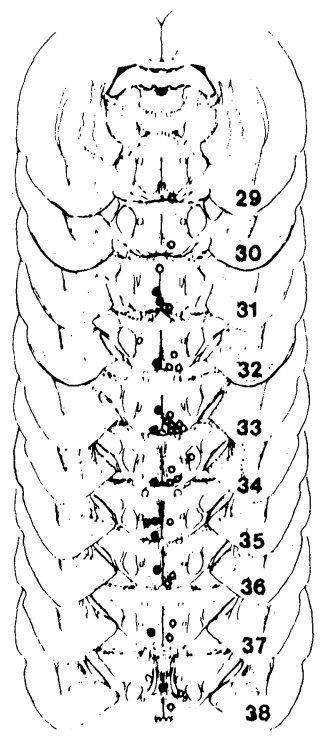

the VMH nuclei, but not elsewhere in the hypothalamus, can suppress feeding. In addition, our data indicate that such implants are equally effective in males and females. Thus, the well established finding that peripheral EB treatment more effectively reduces feeding in females than it does in males, does not seem to result from sex differences in the response of estrogenic mechanisms in the VMH.

\section{REFERENCES}

Beatty, W. W., Powley, T. L., \& Keesey, R. E. Effects of neonatal testosterone injection and hormone replacement in adulthood on body weight and body fat in female rats. Physiology \& Behavior, 1970, 5, 1093-1098.

Bell, D. D., \& Zucker, I. Sex differences in body weight and eating: Organization and and activation by gonadal hormones in the rat. Physiology \& Behavior, 1971, 7, 27-34.

Flerko, B., Mess, B., \& Illei-Donhoffer, A. On the mechanism of androgen sterilization. Neuroendocrinology, 1969, 4, 164-169.

König, J. F. R., \& Klippel, R. A. The rat brain: A stereotaxic atlas of the forebrain and lower parts of the brain stem. Baltim ore: Williams \& Wilkins, 1963.

Nadler, $R$. Intrahypothalamic exploration of androgen-sensitive brain loci in neonatal female rats. Transactions of the New York Academy of Sciences, Series II, 1972, 34, 572-581.

O'Briant, D. A. Central control of feeding by gonadal hormones. Unpublished Masters thesis, North Dakota State University, 1974.

Wade, G. N., \& Zucker, I. Modulation of food intake and locomotor activity in female rats by diencephalic hormone implants. Journal of Comparative \& Physiological Psychology, $1970,72,328-336$

Fig. 1. Location of positive sites where EB suppressed feeding (filled circles) and negative sites (open circles). Although all placements were in the right hemisphere, the positive sites are shown on the left side for greater clarity. Numbers on the sections refer to plates in the König and Klippel (1963) atlas.

Zucker, I. Hormonal determinants of sex differences in saccharin preference, food intake and body weight. Physiology \& Behavior, 1969, 4, 595-602.

(Received for publication February 19, 1974.) 